\title{
IDENTIFICAÇÃO DE ANTOCIANIDINAS EM ACEROLAS DO BANCO ATIVO DE GERMOPLASMA DA UNIVERSIDADE FEDERAL RURAL DE PERNAMBUCO ${ }^{1}$
}

\author{
Vera Lúcia Arroxelas Galvão de LIMA,*, Irapuan Oliveira PINHEIRO³, Márcia Silva do NASCIMENTO4, \\ Patrícia Bezerra GOMES ${ }^{4}$, Nonete Barbosa GUERRA ${ }^{5}$
}

\begin{abstract}
RESUMO
As antocianinas, pigmentos responsáveis pela cor vermelha da acerola madura, foram extraídas e purificadas de 12 genótipos cultivados no Banco Ativo de Germoplasma da Universidade Federal Rural de Pernambuco, com o objetivo de determinar a sua composição. As antocianinas e suas respectivas agliconas, obtidas pela hidrólise ácida, foram separadas por cromatografia líquida de alta eficiência (CLAE) usando uma coluna de fase reversa $\left(C_{18}\right)$. A identificação foi realizada pela ordem de eluição e pelos tempos de retenção dos padrões de referência e das antocianidinas obtidas por hidrólise ácida de uvas Isabel, Patrícia e Red Globe, ameixa, cebola roxa, morango e da casca de manga Tommy Atkins. Os cromatogramas obtidos demonstraram que o perfil antociânico dos genótipos de acerola é relativamente simples, apresentando de três a cinco picos comuns entre eles. Após hidrólise ácida foram obtidas somente duas agliconas identificadas como cianidina e pelargonidina. Comparando os cromatogramas das antocianinas e das antocianidinas e, avaliando os tempos de retenção, foi constatada a presença de antocianinas com diferentes graus de glicosilação e ausência de ácidos acilados em suas moléculas e que as agliconas identificadas, cianidina e pelargonidina, encontravam-se em diferentes proporções nos genótipos estudados.
\end{abstract}

Palavras-chave: antocianidinas, Malpighia emarginata D.C., genótipos, cromatografia líquida de alta eficiência.

\section{SUMMARY}

ANTHOCYANIDINS IDENTIFICATION OF ACEROLA FRUITS FROM ACTIVE GERMOPLASM BANK OF THE RURAL FEDERAL UNIVERSITY OF PERNAMBUCO. The red color of mature acerola is due to the presence of anthocyanins. These pigments were extracted and purified from 12 genotypes cultivated at the Active Germoplasm Bank of the Rural Federal University of Pernambuco in order to determine their composition. The aglycon forms of the anthocyanins were revealed by acid hydrolysis. Anthocyanins and anthocyanidins were separated by using high-performance liquid chromatography (HPLC) using a $\mathrm{C}_{18}$ reversed phase analytical column. Anthocyanidins were identified by comparing HPLC elution order and retention times relative to authentic standards and to anthocyanidins obtained from acid hydrolysis of Isabel, Patrícia and Red Globe grapes, plum, red onion, strawberry and Tommy Atkins mango skin. The chromatograms showed that the acerola genotype anthocyanins profile is simple, showing three to five peaks, similar between them. After the acid hydrolysis, the chromatograms revealed the presence of two aglycons which were identified as cyanidin and pelargonidin. By comparing chromatograms of anthocyanins and anthocyanidins and analyzing the retention times, the presence of anthocyanins with different glycosylation patterns and the absence of acylation in their molecules could be clearly seen. The aglycons identified (cyanidin and pelargonidin) were found in different proportions in these acerola genotypes.

Keywords: anthocyanidins, Malpighia emarginata D.C., genotypes, high performance liquid chromatography.

\section{1 - INTRODUÇÃO}

As antocianinas são pigmentos fenólicos solúveis em água, pertencentes à classe dos flavonóides [5, 30], responsáveis pelas várias nuanças entre laranja, vermelho e azul, exibidas pelas frutas, hortaliças, flores, folhas e raízes [24]. Esses compostos são glicosídeos que, por hidrólise ácida, liberam agliconas também denominadas de antocianidinas e, dentre as encontradas na natureza, apenas seis estão

\footnotetext{
${ }^{1}$ Recebido para publicação em 25/4/2006. Aceito para publicação em 20/10/2006 (001732)

2Departamento de Ciências Domésticas,

Universidade Federal Rural de Pernambuco,

Av. Dom Manoel de Medeiros, s/n, Dois Irmãos, CEP 52171-900,

Recife (PE), Brasil

E-mail:veraarroxelas@hotmail.com

${ }^{3}$ Departamento de Ciências Fisiológicas,

Universidade de Pernambuco, Rua Arnóbio Marques, 310, Santo Amaro, Recife (PE), CEP 50100-130

${ }^{4}$ Departamento de Antibióticos, Universidade Federal de Pernambuco, Av. Morais Rego, 1235, Cidade Universitária, CEP 50670-901, Recife (PE) ${ }^{5}$ Departamento de Nutrição, Universidade Federal de Pernambuco * A quem a correspondência deve ser enviada
}

presentes em alimentos: pelargonidina, cianidina, delfinidina, peonidina, petunidina e malvidina, que diferem entre si quanto ao número de hidroxilas e grau de metilação, presentes no anel B [24].

Nos últimos anos, o interesse por esses pigmentos se intensificou uma vez que pesquisas têm demonstrado que as antocianinas e suas respectivas agliconas são compostos bioativos e que, entre os vários outros efeitos fisiológicos, possuem capacidade antioxidante [19] e propriedade antiinflamatória [32], promovem vaso-dilatação [6], atuam na prevenção da hiperglicemia [25], estimulam a secreção de insulina [18], melhoram a adaptação da visão noturna e previnem a fadiga visual [27]. Recentemente, ZHANG, VAREED \& NAIR [40] constataram o efeito inibitório da cianidina, delfinidina, pelargonidina, petunidina e malvidina na proliferação de células humanas cancerígenas, originadas em diferentes partes do corpo: estômago, cólon, mama, pulmão e sistema nervoso central.

Desta forma, é importante conhecer a distribuição e a estrutura química das antocianinas em alimentos. As técnicas de separação e identificação mais utilizadas estão 
baseadas em equipamentos sofisticados e, conseqüentemente, onerosos como o cromatógrafo líquido de alta eficiência (CLAE), utilizando coluna de fase reversa para separação e identificação por ressonância magnética nuclear [13], arranjo de diodos e espectrometria de massa [7, 14] e eletroforese de zona de capilaridade [17].

Entretanto, para identificar e quantificar as antocianinas, individualmente, por meio da CLAE de fase reversa, o maior desafio é a disponibilidade dos padróes de referência para mais de 600 compostos já identificados, conforme WROLSTAD [37], ao se referir à apresentação de ANDERSEN [2], durante o Workshop Internacional de Antocianinas. Esta diversidade é resultante do número de grupos hidroxila e metoxila, da natureza e do número de açúcares e de ácidos alifáticos e/ou aromáticos, bem como da localização desses compostos presentes na aglicona [35]. No entanto, por hidrólise ácida, o número de antocianinas pode ser reduzido gerando estruturas menos complexas, as antocianidinas, cujos padrões encontram-se comercialmente disponíveis [28].

Tendo em vista que as antocianinas são os pigmentos responsáveis pela cor vermelha da acerola madura [21] e considerando os benefícios que suas agliconas podem proporcionar à saúde humana além da escassez de estudos voltados para esses compostos em acerola, este trabalho teve como objetivo identificar as antocianidinas em frutos de 12 genótipos de acerola do Banco Ativo de Germoplasma da Universidade Federal Rural de Pernambuco.

\section{2 - MATERIAL E MÉTODOS}

\section{1 - Frutos}

As acerolas foram colhidas de 12 genótipos, identificados com os códigos 002-SPE, 003-APE, 004-RPE, 005-APE, 006-TPA, 007-TPA, 008-CPA, 011-BPA, 012-CPA, 013-CPA, 014-CPA e 015-CPA, cultivados no Banco Ativo de Germoplasma da UFRPE, instalado na Estação Experimental de Cana-de-Açúcar em Carpina, município do Estado de Pernambuco. Frutos no estádio maduro ( $1 \mathrm{~kg}$ ) foram colhidos de cada genótipo no período da manhã, refrigerados e transportados, em caixa isotérmica, até o Laboratório de Experimentação e Análises de Alimentos (LEAAL) "Nonete Barbosa Guerra" do Departamento de Nutrição da UFPE, onde foram realizados os experimentos. Após a seleção, com o objetivo de obter frutos em estádio de maturação, com coloração característica de cada genótipo, eles foram processados em centrífuga doméstica e as polpas resultantes, imediatamente armazenadas sob congelamento $\left(-18 \pm 1{ }^{\circ} \mathrm{C}\right)$. Todos os procedimentos subseqüentes foram realizados sob reduzida luminosidade.

\section{2 - Extração das antocianinas}

As antocianinas da polpa de acerola, de cada genótipo, foram extraídas com acetona, filtradas a vácuo e o resíduo reextraído com acetona até a completa remoção dos pig- mentos. O volume do filtrado foi quantificado, transferido para um funil de separação, ao qual foi adicionado o dobro do volume de clorofórmio, e estocado durante uma noite a $4{ }^{\circ} \mathrm{C}$, para a separação das duas fases. A fase aquosa (fase superior) foi levada ao evaporador rotativo $\left(38 \pm 2{ }^{\circ} \mathrm{C}\right) \mathrm{du}$ rante 10-15 minutos para a remoção da acetona residual e o extrato aquoso, estocado a $-18{ }^{\circ} \mathrm{C}$ para ser utilizado nas etapas posteriores [31].

\section{3 - Purificação e precipitação das antocianinas}

As antocianinas foram isoladas pela técnica de extração em fase sólida utilizando cartuchos Sep-Pak $\mathrm{C}_{18}$ (Waters Associates, Milford, MA), conforme a metodologia descrita por RODRIGUEZ-SAONA \& WROLSTAD [31]. Esta técnica propicia a remoção de açúcares, de ácidos e de outros compostos fenólicos das amostras. O extrato metanólico obtido após a purificação foi concentrado sob temperatura controlada $\left(38 \pm 2{ }^{\circ} \mathrm{C}\right.$ ) e pressão reduzida até a obtenção de um resíduo seco ao qual foi adicionado éter dietílico com a finalidade de recuperar os pigmentos aderidos à parede do balão sem haver sua dissolução. As antocianinas foram separadas por centrifugação (20 min, 2500 x g) e secas sob atmosfera de nitrogênio. Os pigmentos em pó foram acondicionados em vidros âmbar que receberam fluxo de nitrogênio, antes de serem fechados, e, em seguida, armazenados sob refrigeração $\left(4{ }^{\circ} \mathrm{C}\right)$, até o momento das análises [11].

\section{4 - Preparação das antocianinas}

Antocianinas purificadas de cada genótipo ( $1 \mathrm{mg}$ ), dissolvidas em $2-3$ gotas de metanol $(0,01 \% \mathrm{HCl})$, com posterior adição de $1 \mathrm{~mL}$ de ácido fosfórico 4\% (água Milli-Q), foram filtradas antes de serem injetadas no cromatógrafo em membranas Millipore tipo HV (Millipore Corp. Bedford, MA) com poro de $0,45 \mu \mathrm{m}$ e $13 \mathrm{~mm}$ de diâmetro.

\section{5 - Hidrólise ácida das antocianinas}

A hidrólise ácida das antocianinas foi realizada conforme descrito por DRUST \& WROLSTAD [10], com algumas modificações. Em tubos de vidro rosqueados, cerca de 3 a $5 \mathrm{mg}$ de antocianinas purificadas de cada genótipo foram dissolvidas em $2-3$ gotas de metanol $(0,01 \% \mathrm{HCl})$, adicionados $10 \mathrm{~mL}$ de $\mathrm{HCl} 2 \mathrm{M}$ e fechados, após aplicação de fluxo de gás nitrogênio. Os pigmentos foram hidrolisados por 60 min em banho de água fervente e, então, imediatamente resfriados em banho de gelo. O hidrolisado foi purificado pela extração em fase sólida utilizando cartuchos Sep-Pak $\mathrm{C}_{18}$ (Waters), previamente ativados com metanol $(0,01 \%$ $\mathrm{HCl})$ seguido por água acidificada $(0,01 \% \mathrm{HCl})$. As amostras foram aplicadas nos cartuchos, lavadas com $10 \mathrm{~mL}$ de água acidificada $(0,01 \% \mathrm{HCl})$ e as antocianidinas, eluídas com $5 \mathrm{~mL}$ de metanol acidificado $(0,01 \% \mathrm{HCl})$. Este volume foi coletado e conduzido a um evaporador rotativo $\left(38 \pm 2{ }^{\circ} \mathrm{C}\right.$, sob vácuo) até que o metanol fosse removido próximo à secura. Antes de serem injetadas no cromatógrafo, as an- 
tocianidinas foram dissolvidas com $1 \mathrm{~mL}$ de ácido fosfórico 4\% (água Milli-Q) e filtradas em membranas Millipore tipo HV (Millipore Corp. Bedford, MA), de poro de 0,45 $\mu \mathrm{m}$ e 13 mm de diâmetro.

\section{6 - Separação das antocianinas e das antocianidinas}

As antocianinas e antocianidinas foram separadas por cromatografia líquida de alta eficiência segundo metodologia descrita por DRUST \& WROLSTAD [10]. Esta etapa foi realizada no Laboratório de Produtos Naturais do Departamento de Antibióticos/UFPE, utilizando um cromatógrafo analítico Hewlett-Packard série 1100, equipado com desgaseificador, bomba quaternária, forno e detectores de ultravioleta-visível e de índice de refração. Todo o cromatógrafo foi controlado através do software Chemstation, também responsável pela obtenção e tratamento dos dados, instalado em um computador Kayak Hewllet-Packard com o sistema operacional Windows NT. A separação foi realizada com uma coluna de fase reversa $\mathrm{C}_{18}$ (Merck, Lichrospher 100RP-18, Darmstadt, Alemanha) de $250 \mathrm{~mm}$ de comprimento, $4 \mathrm{~mm}$ de diâmetro interno e com partículas de $5 \mu \mathrm{m}$ de diâmetro médio, acoplada a uma coluna de guarda de $4 \mathrm{~mm}$ de comprimento e $3 \mathrm{~mm}$ diâmetro interno (Merck, Lichrospher 100RP-18, Darmstadt, Alemanha). O volume de injeção foi $20 \mu \mathrm{L}$, o comprimento de onda $520 \mathrm{~nm} \mathrm{e}$, a temperatura e a vazão, controladas em $30{ }^{\circ} \mathrm{C}$ e $1 \mathrm{~mL} / \mathrm{min}$, respectivamente. Para eluição, foi utilizada uma alimentação em gradiente formada a partir de duas soluções: solvente A (100\% acetonitrila, grau HPLC) e solvente B ( $1 \%$ ácido fosfórico, $10 \%$ ácido acético e $5 \%$ acetonitrila (v/v), em água Milli-Q). A eluição seguiu um gradiente linear variando de 5 a $20 \%$ a participação do solvente A na composição da fase móvel num intervalo de 20 min e, então, um gradiente linear para este mesmo solvente de 20 a $5 \%$ durante 5 min, retornando à composição inicial da fase móvel ( $5 \%$ solvente A e $95 \%$ solvente B).

\section{7 - Identificação das antocianidinas}

A identificação das antocianidinas foi realizada a partir da comparação dos tempos de retenção obtidos nos cromatogramas das amostras, com os tempos de retenção dos padrões de cloretos de cianidina, pelargonidina e malvidina (Extrasynthese, Genay, França). Também foi realizada uma comparação com os tempos de retenção obtidos das antocianidinas de uvas Isabel, Patrícia e Red Globe, ameixa, cebola roxa, morango e da casca de manga Tommy Atkins [9, 3, 38], adquiridos no mercado local, com a finalidade de se obter o perfil cromatográfico das seis antocianidinas. As análises seguiram os procedimentos anteriormente descritos.

\section{3 - RESULTADOS E DISCUSSÃo}

Os perfis antociânicos das polpas dos diferentes genótipos de acerola são relativamente simples quando comparados aos de frutos como "lowbush blueberries" (Vaccinium angustifolium Ait., cv. 'Fundy'), nos quais foram identificadas pelo menos 25 antocianinas [12] e aos extratos de "bilberry" (Vaccinium myrtillus L.), nos quais ZHANG et al. [39] encontraram mais de 15 picos referentes à presença de antocianinas que, após a hidrólise ácida, foram reduzidos a cinco, identificados como delfinidina, cianidina, petunidina, peonidina e malvidina.

No que diz respeito às antocianinas das acerolas (Figuras 1-6), verifica-se que os genótipos 002-SPE, 004-RPE, 005-APE, 006-TPA, 011-BPA, 013-CPA e 014-CPA apresentaram três picos similares, enquanto os 003-APE, 007-TPA, 008-CPA e 015-CPA, apresentaram quatro picos com o mesmo perfil e o 012-CPA que, além dos picos comuns aos demais, apresentou mais um. Tendo como base a área dos picos, constata-se que o registrado em menor tempo de retenção, em todos os cromatogramas, encontrava-se, provavelmente, presente em maior quantidade.

Convém ressaltar que o tempo de retenção, parâmetro baseado na hidrofobicidade da molécula, é influenciado pelo grau de glicosilação e pela natureza dos açúcares presentes nesses pigmentos. CHAOVANALIKIT, THOMPSON \& WROLSTAD [7], em frutos de "blue honeysuckle" (Lonicera caerulea L.), relataram que seis antocianinas foram separadas por CLAE, utilizando coluna de fase reversa na seguinte ordem de eluição: cianidina 3,5-diglicosídeo, cianidina 3-glicosídeo, cianidina 3-rutinosídeo, pelargonidina 3-glicosídeo, peonidina 3-glicosídeo, peonidina 3-rutinosídeo.

De acordo com a metodologia descrita por DRUST \& WROLSTAD [10], o tempo de eluição, indicado para separação de antocianinas aciladas por CLAE, utilizando coluna $\mathrm{C}_{18}$ de fase reversa $(5 \mu \mathrm{m} \times 250 \mathrm{~mm} \times 4,6 \mathrm{~mm})$, é de $70 \mathrm{~min}$. Este tempo prolongado é devido à presença de ácidos acilados na molécula que aumentam sua afinidade com a fase estacionária, ou seja, aumentam o tempo de retenção [35]. Como nos cromatogramas obtidos nesta pesquisa, os picos foram registrados antes de $15 \mathrm{~min}$, infere-se que esses pigmentos não possuem ácidos em suas moléculas. Este resultado corrobora os obtidos por HANAMURA, HAGIWARA \& KAWAGISHI [15], os quais relataram, em acerolas cedidas pela Nichirei do Brazil (Recife, Brasil), a ausência de acilação nas moléculas das antocianinas identificadas.

Pesquisas realizadas por LONGO \& VASAPOLLO [23] e por KALLITHRAKA et al. [20], em frutos de Ruscus aculeatus L. e em uvas, respectivamente, mostraram que os picos referentes às antocianinas aciladas foram os últimos a serem eluídos e que, nas uvas, os picos referentes aos glicosídeos de delfinidina, cianidina, petunidina e malvidina foram eluídos antes de $13 \mathrm{~min}$, enquanto o pico referente a malvidina, acilada com ácido $p$-cumárico, foi registrado no cromatograma, aproximadamente, aos 22 min de eluição.

Diante da impossibilidade de obter padrões que permitissem a identificação das antocianinas, foi procedida a hidrólise ácida para obtenção das antocianidinas. Embora DRUST \& WROLSTAD [10] recomendem 30 min para hidrólise ácida, neste estudo foram utilizados $60 \mathrm{~min}$, uma vez que, em experimentos implementados por ZHANG et al. [39], foi constatado, após $30 \mathrm{~min}$ a $100 \pm 2{ }^{\circ} \mathrm{C}$, a presença de $10 \%$ de antocianinas, cujo desaparecimento 
quase completo ocorreu somente após 60 min de hidrólise. Posteriormente, LONGO, VASAPOLLO \& RESCIO [22] constataram que este tempo também não foi suficiente para hidrolisar totalmente as antocianinas purificadas de "buckthorn" (Rhamnus alaternus L), cuja hidrólise total foi obtida apenas com cinco h.

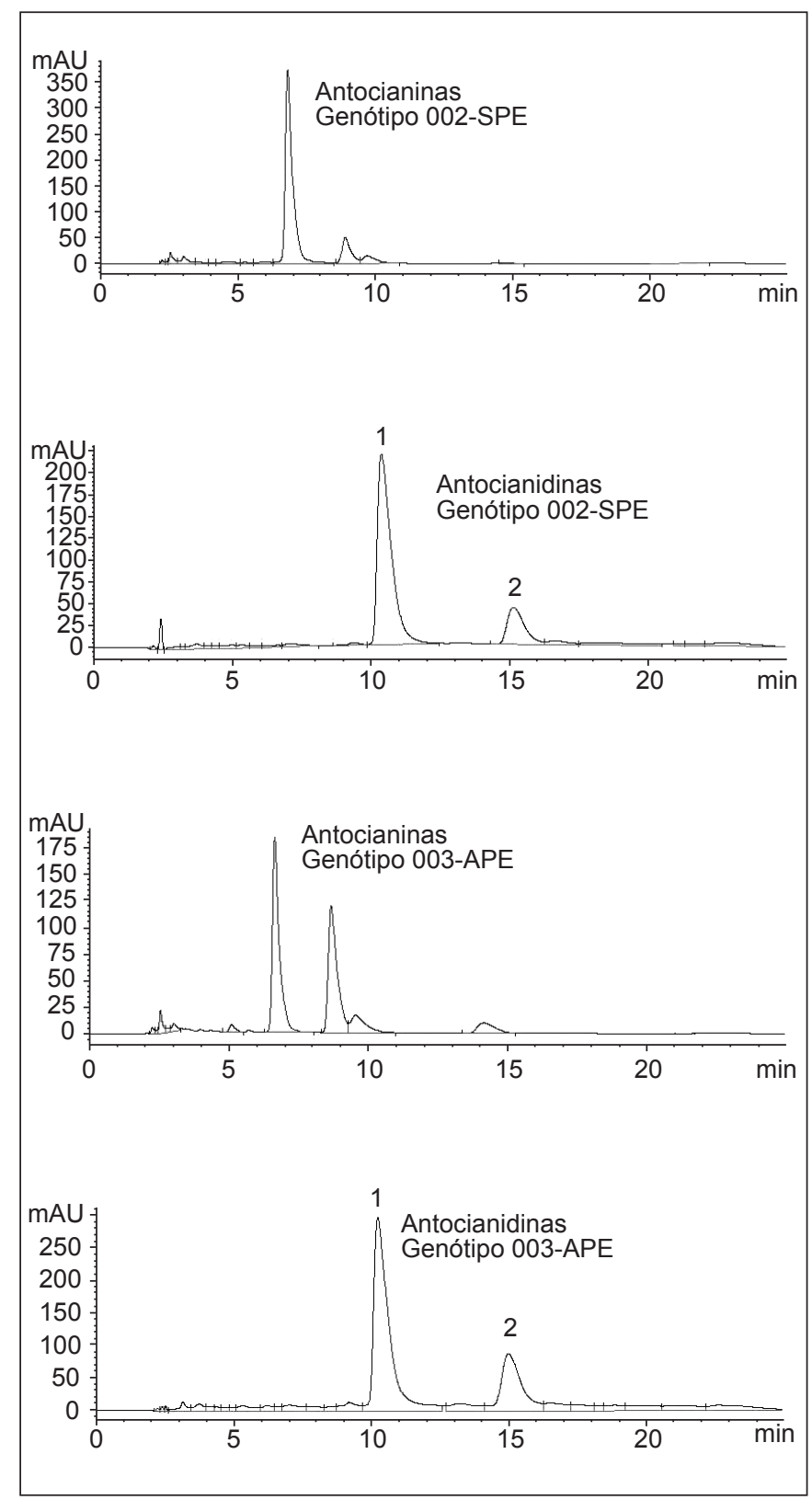

FIGURA 1 - Perfil cromatográfico obtido por CLAE das antocianinas e antocianidinas presentes em genótipos de acerola. Condições cromatográficas: coluna de fase reversa $\mathrm{C}_{18}$ Lichrospher 100RP-18 (250 x $4 \mathrm{~mm})$, acoplada a uma coluna de guarda Lichrospher 100RP-18 (4 x $3 \mathrm{~mm})$. Solvente A: $100 \%$ acetonitrila (grau HPLC); solvente B: 1\% ácido fosfórico, 10\% ácido acético e $5 \%$ acetonitrila (v/v, em água Milli-Q). Gradiente linear de 5 a $20 \%$ do solvente A em 20 min e de 20 a $5 \%$ em 5 min, retornando à composição inicial da fase móvel $(5 \%$ solvente A e $95 \%$ solvente B). Temperatura de $30{ }^{\circ} \mathrm{C}$, vazão de $1 \mathrm{~mL} / \mathrm{min}$, volume de injeção de $20 \mu \mathrm{L}$ e deteç̧ão em 520 nm. Pico 1: cianidina; Pico 2: pelargonidina.
Comparando os cromatogramas, antes e após a hidrólise, pode-se constatar que, para o fruto em estudo, o tempo utilizado foi efetivo para obter apenas as antocianidinas. Também permitiu confirmar a presença de pigmentos glicosilados na medida em que os picos presentes nos cromatogramas das antocianinas desapareceram após a hidrólise

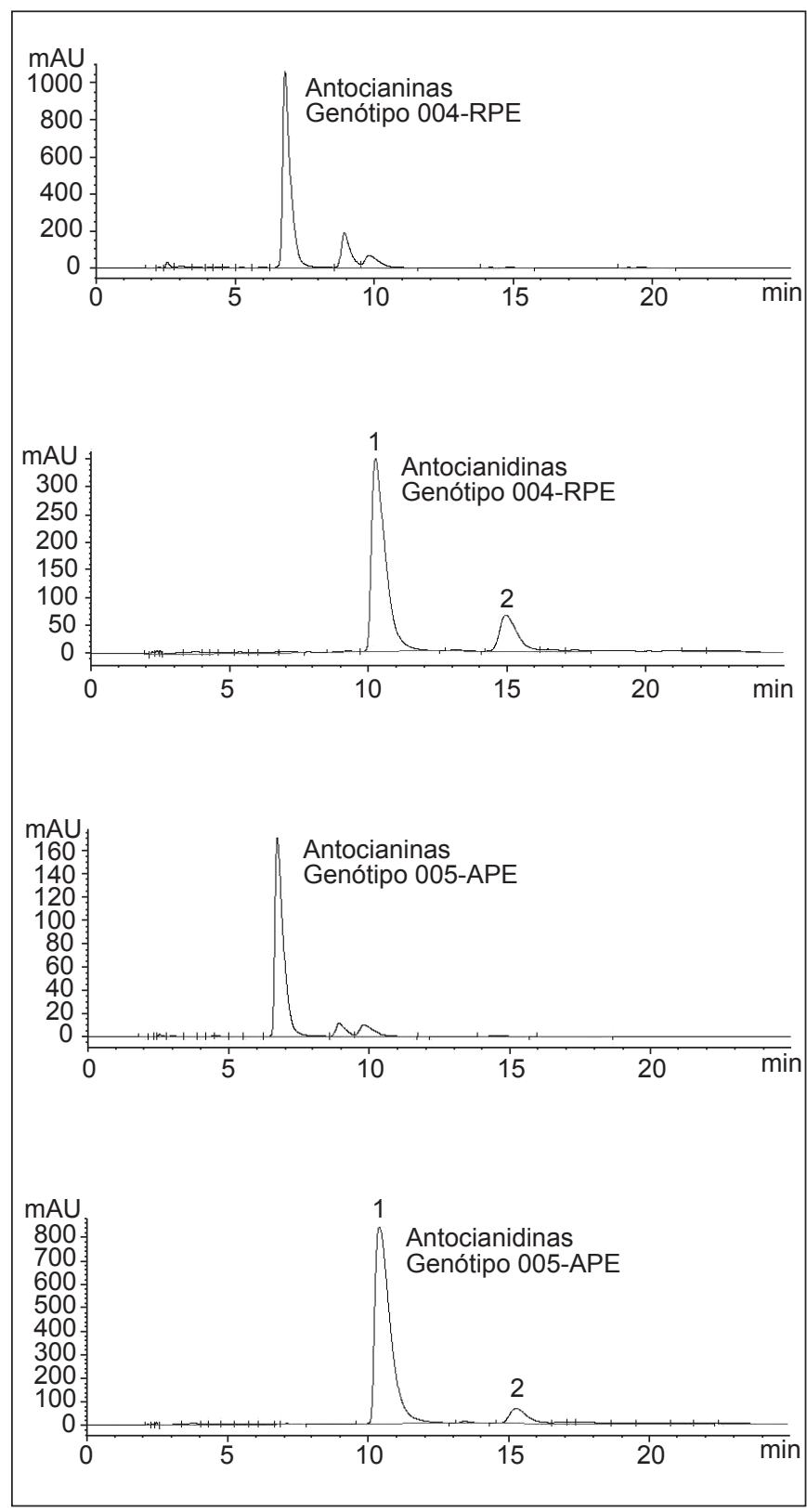

FIGURA 2 - Perfil cromatográfico obtido por CLAE das antocianinas e antocianidinas presentes em genótipos de acerola. Condições cromatográficas: coluna de fase reversa $\mathrm{C}_{18}$ Lichrospher 100RP-18 (250 x $4 \mathrm{~mm})$, acoplada a uma coluna de guarda Lichrospher 100RP-18 (4 x $3 \mathrm{~mm}$ ). Solvente A: 100\% acetonitrila (grau HPLC); solvente B: $1 \%$ ácido fosfórico, $10 \%$ ácido acético e $5 \%$ acetonitrila (v/v, em água Milli-Q). Gradiente linear de 5 a $20 \%$ do solvente A em 20 min e de 20 a $5 \%$ em 5 min, retornando à composição inicial da fase móvel $(5 \%$ solvente $\mathrm{A}$ e $95 \%$ solvente B). Temperatura de $30{ }^{\circ} \mathrm{C}$, vazão de $1 \mathrm{~mL} / \mathrm{min}$, volume de injeção de $20 \mu \mathrm{L}$ e detecção em $520 \mathrm{~nm}$. Pico 1: cianidina; Pico 2: pelargonidina 


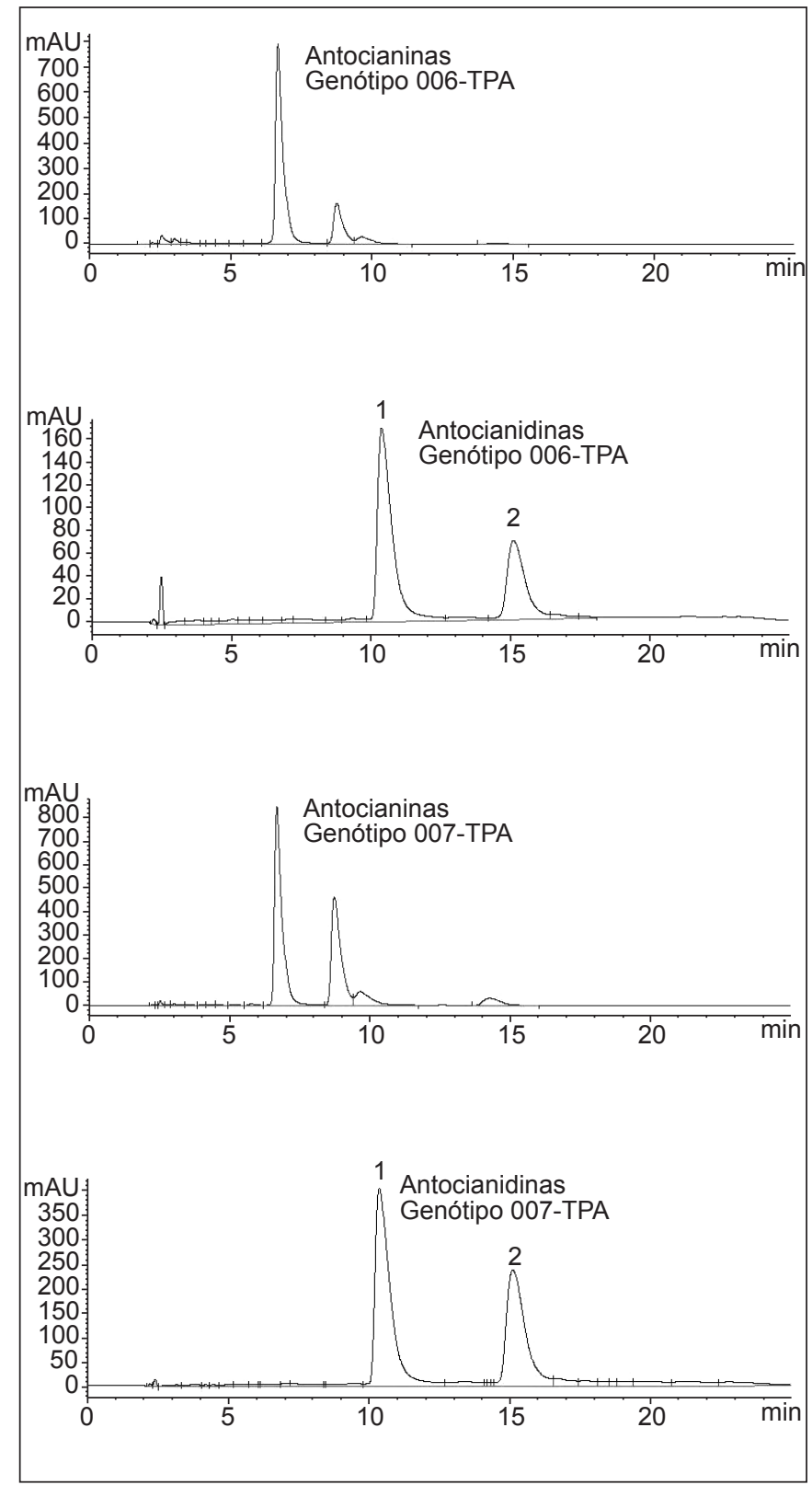

FIGURA 3 - Perfil cromatográfico obtido por CLAE das antocianinas e antocianidinas presentes em genótipos de acerola. Condições cromatográficas: coluna de fase reversa $\mathrm{C}_{18}$ Lichrospher 100RP$18(250 \times 4 \mathrm{~mm})$, acoplada a uma coluna de guarda Lichrospher 100RP-18 (4 x $3 \mathrm{~mm}$ ). Solvente A: $100 \%$ acetonitrila (grau HPLC); solvente B: $1 \%$ ácido fosfórico, $10 \%$ ácido acético e $5 \%$ acetonitrila (v/v, em água Milli-Q). Gradiente linear de 5 a $20 \%$ do solvente A em 20 min e de 20 a $5 \%$ em 5 min, retornando à composição inicial da fase móvel (5\% solvente A e $95 \%$ solvente B). Temperatura de $30^{\circ} \mathrm{C}$, vazão de $1 \mathrm{~mL} / \mathrm{min}$, volume de injeção de $20 \mu \mathrm{L}$ e detecção em 520 nm. Pico 1: cianidina; Pico 2: pelargonidina.

ácida dando origem, nos cromatogramas das agliconas, a novos picos, de maior intensidade e de diferentes tempos de retenção (Figuras 1-6).

A natureza e o grau de substituição no anel B das agliconas influenciam no tempo de retenção, tendo em vista

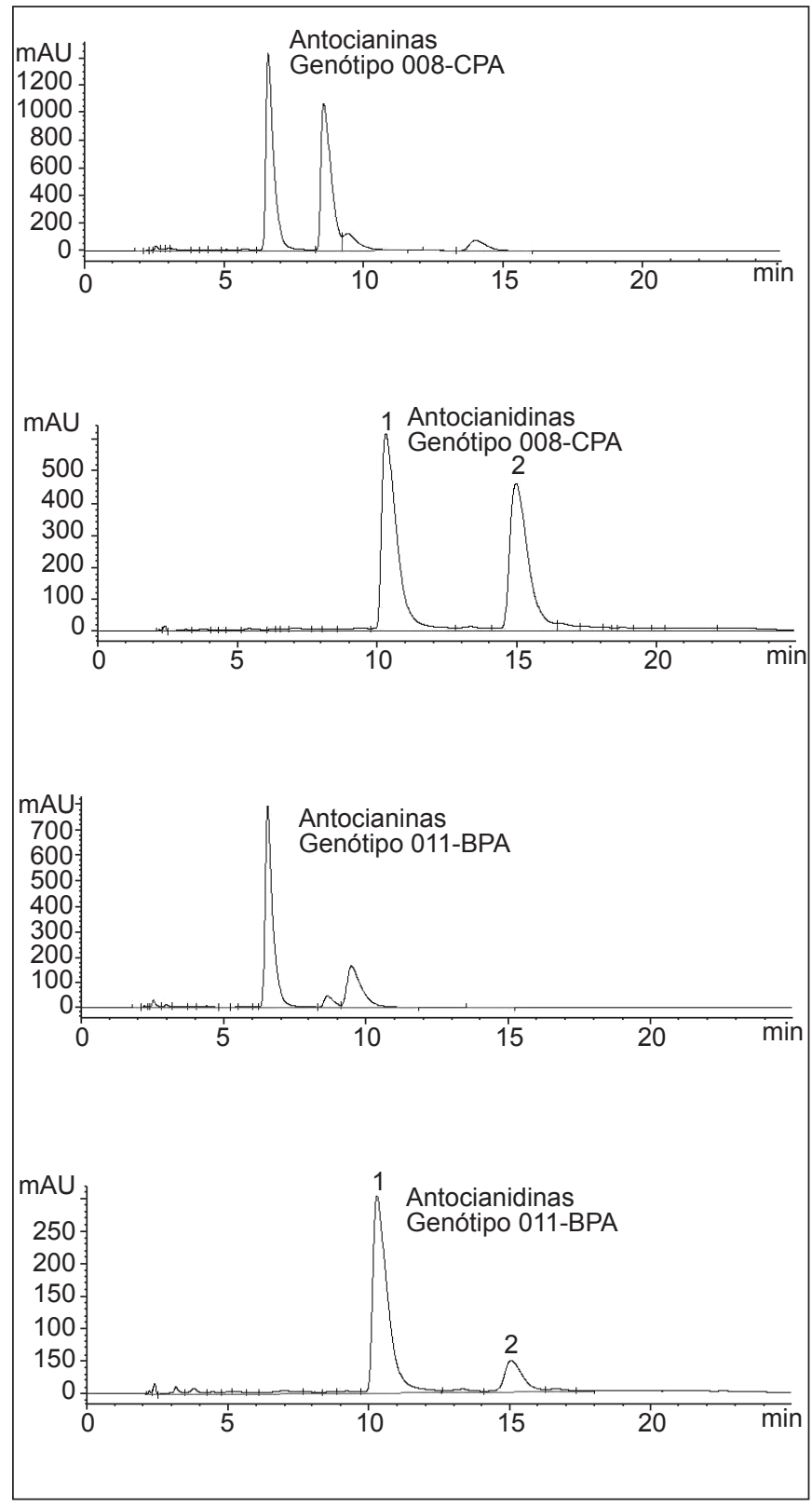

FIGURA 4 - Perfil cromatográfico obtido por CLAE das antocianinas e antocianidinas presentes em genótipos de acerola. Condições cromatográficas: coluna de fase reversa $\mathrm{C}_{18}$ Lichrospher 100RP-18 (250 x $4 \mathrm{~mm})$, acoplada a uma coluna de guarda Lichrospher 100RP-18 (4 x 3 mm). Solvente A: 100\% acetonitrila (grau HPLC); solvente B: $1 \%$ ácido fosfórico, $10 \%$ ácido acético e $5 \%$ acetonitrila (v/v, em água Milli-Q). Gradiente linear de 5 a $20 \%$ do solvente A em 20 min e de 20 a $5 \%$ em 5 min, retornando à composição inicial da fase móvel $(5 \%$ solvente $\mathrm{A}$ e $95 \%$ solvente B). Temperatura de $30{ }^{\circ} \mathrm{C}$, vazão de $1 \mathrm{~mL} / \mathrm{min}$, volume de injeção de $20 \mu \mathrm{L}$ e detecção em $520 \mathrm{~nm}$. Pico 1: cianidina; Pico 2: pelargonidina.

que a presença de grupos hidroxilas aumenta a mobilidade da molécula, enquanto a de grupos metoxilas, decresce; características que, em análise realizada em CLAE de fase reversa, estabelecem a ordem de eluição das antocianidinas: delfinidina, cianidina, petunidina, pelargonidina, peonidina 


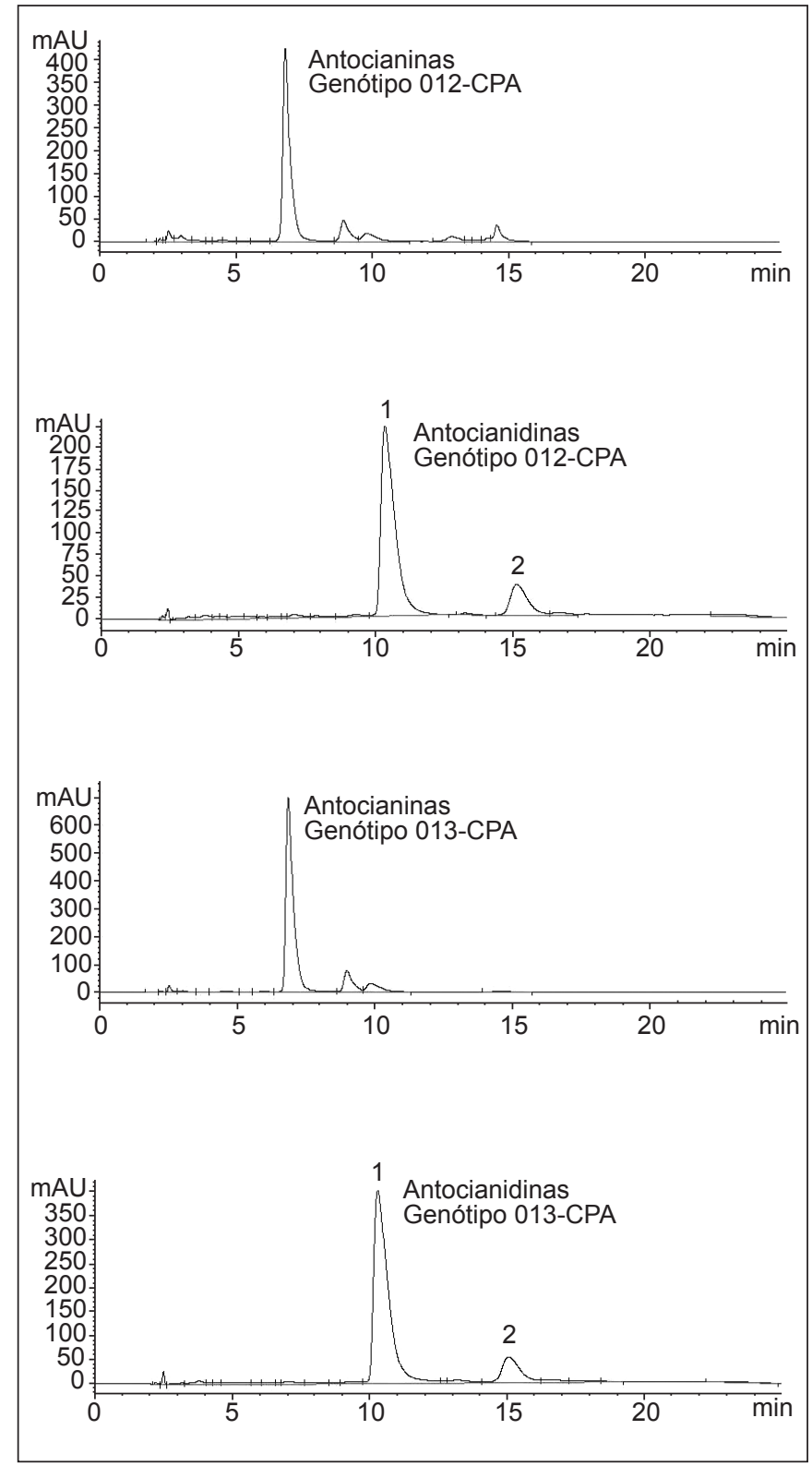

FIGURA 5 - Perfil cromatográfico obtido por CLAE das antocianinas e antocianidinas presentes em genótipos de acerola. Condições cromatográficas: coluna de fase reversa $\mathrm{C}_{18}$ Lichrospher 100RP-18 (250 x $4 \mathrm{~mm}$ ), acoplada a uma coluna de guarda Lichrospher 100RP-18 (4 x $3 \mathrm{~mm}$ ). Solvente A: 100\% acetonitrila (grau HPLC); solvente B: $1 \%$ ácido fosfórico, $10 \%$ ácido acético e $5 \%$ acetonitrila (v/v, em água Milli-Q). Gradiente linear de 5 a $20 \%$ do solvente A em 20 min e de 20 a $5 \%$ em 5 min, retornando à composição inicial da fase móvel $(5 \%$ solvente $\mathrm{A}$ e $95 \%$ solvente B). Temperatura de $30{ }^{\circ} \mathrm{C}$, vazão de $1 \mathrm{~mL} / \mathrm{min}$, volume de injeção de $20 \mu \mathrm{L}$ e detecção em $520 \mathrm{~nm}$. Pico 1: cianidina; Pico 2: pelargonidina.

e malvidina [8,35]. Considerando a ordem de eluição e os tempos de retenção exibidos nos cromatogramas dos padrões (Figura 7) e dos vegetais citados em 2.9 (Tabela 1), as duas agliconas presentes em todos os genótipos de acerola em

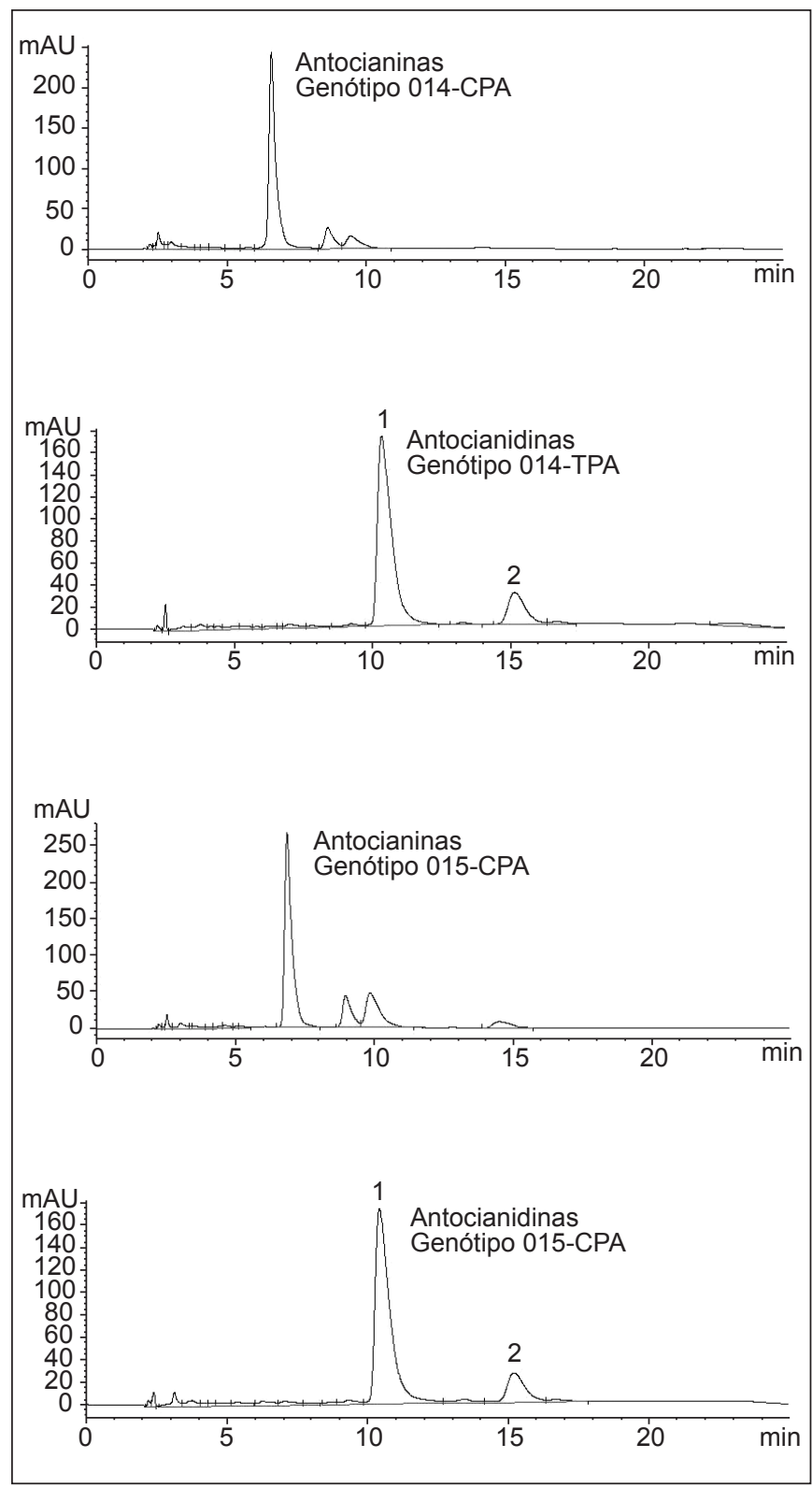

FIGURA 6 - Perfil cromatográfico obtido por CLAE das antocianinas e antocianidinas presentes em genótipos de acerola. Condições cromatográficas: coluna de fase reversa $\mathrm{C}_{18}$ Lichrospher 100RP-18 (250 x $4 \mathrm{~mm})$, acoplada a uma coluna de guarda Lichrospher 100RP-18 (4 x $3 \mathrm{~mm})$. Solvente A: 100\% acetonitrila (grau HPLC); solvente B: $1 \%$ ácido fosfórico, $10 \%$ ácido acético e $5 \%$ acetonitrila (v/v, em água Milli-Q). Gradiente linear de 5 a $20 \%$ do solvente A em 20 min e de 20 a $5 \%$ em 5 min. retornando à composição inicial da fase móvel $(5 \%$ solvente $\mathrm{A}$ e $95 \%$ solvente B). Temperatura de $30^{\circ} \mathrm{C}$, vazão de $1 \mathrm{~mL} / \mathrm{min}$, volume de injeção de $20 \mu \mathrm{L}$ e detecção em $520 \mathrm{~nm}$. Pico 1: cianidina; Pico 2: pelargonidina

estudo foram identificadas como cianidina e pelargonidina (Figuras 1-6).

Este achado concorda com MACHEIX, FLEURIET \& BILLOT [24], ao afirmarem que a maioria dos frutos con- 


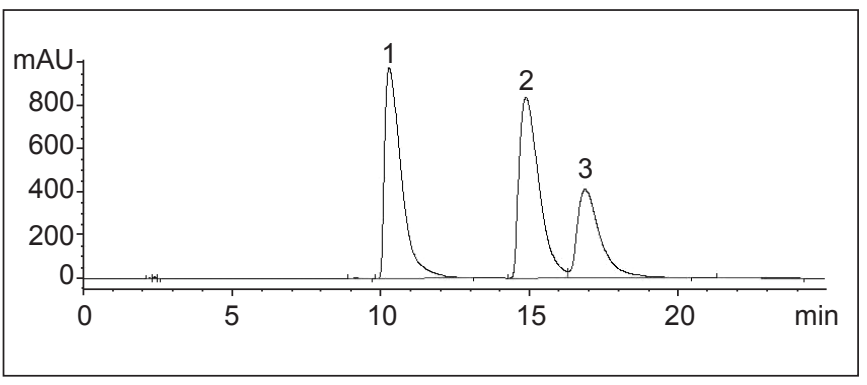

FIGURA 7 - Perfil cromatográfico obtido por CLAE dos padrões de referência. Condições cromatográficas: coluna de fase reversa $\mathrm{C}_{18}$ Lichrospher 100RP-18 (250 x $\left.4 \mathrm{~mm}\right)$, acoplada a uma coluna de guarda Lichrospher 100RP-18 (4 $\times 3 \mathrm{~mm})$. Solvente A: $100 \%$ acetonitrila (grau HPLC); solvente B: $1 \%$ ácido fosfórico, $10 \%$ ácido acético e $5 \%$ acetonitrila (v/v, em água Milli-Q). Gradiente linear de 5 a $20 \%$ do solvente A em 20 min e de 20 a $5 \%$ em 5 min, retornando à composição inicial da fase móvel ( $5 \%$ solvente A e $95 \%$ solvente B). Temperatura de $30^{\circ} \mathrm{C}$, vazão de $1 \mathrm{~mL} / \mathrm{min}$, volume de injeção de $20 \mu \mathrm{L}$ e detecção em $520 \mathrm{~nm}$. Pico 1: cianidina; Pico 2: pelargonidina; Pico 3: malvidina

TABELA 1 - Tempo de retenção e identificação das antocianidinas presentes em diferentes vegetais.

\begin{tabular}{lcccccc}
\hline \multirow{2}{*}{ Fontes Vegetais } & \multicolumn{6}{c}{ Tempo de retenção (min) } \\
\cline { 2 - 7 } & \multicolumn{7}{c}{ Antocianidinas } \\
\cline { 2 - 7 } & $\mathbf{D f}^{*}$ & $\mathbf{C y}$ & $\mathbf{P t}$ & $\mathbf{P g}$ & $\mathbf{P n}$ & $\mathbf{M v}$ \\
\hline Uva Isabel & 6,83 & 10,47 & 11,47 & nd & nd & 16,88 \\
Uva Patrícia & 6,77 & 10,42 & nd & nd & nd & 17,04 \\
Uva Red Globe & 6,45 & 9,94 & nd & nd & 16,15 & nd \\
Ameixa & nd & 10,26 & nd & nd & nd & nd \\
Cebola roxa & nd & 9,60 & nd & nd & 16,00 & nd \\
Morango & nd & 10,40 & nd & 15,16 & nd & nd \\
Manga Tommy Atkins & nd & 10,01 & nd & 15,17 & 16,03 & nd \\
\hline
\end{tabular}

*Df: delfinidina; Cy: cianidina; Pt: petunidina; Pg: pelargonidina; Pn: peonidina; Mv: malvidina; e nd: não detectada.

tém duas antocianidinas, das quais a cianidina é a mais encontrada, acompanhada pela peonidina, delfinidina ou pelargonidina e concorda, também, com HANAMURA, HAGIWARA \& KAWAGISHI [15], ao relatarem que, em acerolas, as antocianinas identificadas foram cianidina 3-ramnosídeo e a pelargonidina 3-ramnosídeo, as mesmas agliconas encontradas neste estudo.

Em pesquisas realizadas por VENDRAMINI \& TRUGO [36] e SILVA [33] em acerolas oriundas de um plantio comercial instalado no Rio de Janeiro (RJ) e em acerolas de quatro diferentes cultivares, provenientes da região de Campinas-SP, respectivamente, além da presença de pelargonidina e cianidina também foi detectada a malvidina. Este resultado pode ser decorrente da diversidade genética gerada pela propagação da aceroleira por sementes [29] e de complexos fatores, como incidência de luz solar, pluviosidade, topografia, tipo de solo, localização geográfica, estação do ano, fertilização do solo, grau de maturação [16] e, ainda, das diferentes técnicas analíticas utilizadas na identificação desses compostos.
Tendo como base a percentagem da área dos picos registrados nos cromatogramas, foi constatado (Tabela 2) que os genótipos possuem diferenciadas proporções das agliconas identificadas, as quais variaram de 53,23 a 90,22\% de cianidina e 9,78 a $46,77 \%$ de pelargonidina. BOYLES \& WROLSTAD [4], em 46 amostras de sucos de framboesa vermelha obtidos de diferentes cultivares, de diferentes locais de origem, de diferentes estádios de maturação e de diferentes métodos de processamento, relataram que, embora não tenham encontrado as mesmas antocianinas, os cromatogramas das antocianidinas revelaram, com diferentes proporções das áreas dos picos, que todas as amostras continham cianidina e pelargonidina.

TABELA 2 - Distribuição relativa das antocianidinas presentes em 12 genótipos de acerola.

\begin{tabular}{ccc}
\hline Genótipos & \multicolumn{2}{c}{ Antocianidinas } \\
& (\% da área do pico registrado a 520 $\mathbf{~ m})$ \\
\cline { 2 - 3 } & Cianidina & Pelargonidina \\
\hline 002-SPE & 81,24 & 18,76 \\
003-APE & 71,59 & 28,41 \\
004-RPE & 80,91 & 19,09 \\
005-APE & 90,22 & 9,78 \\
006-TPA & 66,68 & 33,32 \\
007-TPA & 59,06 & 40,94 \\
008-CPA & 53,23 & 46,77 \\
011-BPA & 84,22 & 15,78 \\
012-CPA & 83,11 & 16,89 \\
013-CPA & 85,67 & 14,33 \\
014-CPA & 82,41 & 17,59 \\
015-CPA & 84,23 & 15,77 \\
\hline
\end{tabular}

Diferenças qualitativas e quantitativas do perfil antociânico também foram relatadas por: ANCOS, GONZÁLES \& CANO [1] em quatro cultivares de framboesa; por KALLITHRAKA et al. [20] em de 17 cultivares de uvas; e por SIRIWOHARN et al. [34] em 11 cultivares de amora preta. MOZET, TREBŠE \& HRIBAR [26], por meio da CLAE, detectaram um perfil semelhante composto por oito antocianinas, com pequena variação na intensidade relativa dos picos em cinco cultivares de cereja doce. Resultados similares foram obtidos por DONNER, GAO \& MAZZA [9] em quatro cultivares de cebola roxa, ao relatarem um perfil cromatográfico qualitativo idêntico, com variação na quantidade relativa de antocianinas demonstrando a complexidade envolvida na determinação destes compostos.

\section{4 - CONCLUSÕES}

O simplificado perfil antociânico apresentado pelos 12 genótipos sugere a ocorrência de diferentes graus de glicosilação e ausência de ácidos acilados nas moléculas das antocianinas e que apenas duas agliconas, cianidina e pelargonidina, em diferenciadas proporções, encontravamse presentes nos genótipos estudados.

\section{5 - REFERÊNCIAS BIBLIOGRÁFICAS}

[1] ANCOS, B.; GONZALES, E.; CANO, M. P. Differentiation of raspberry varieties according to anthocyanin com- 
position. Zeitschrift für Lebensmittel -Untersuchung und -Forschung A, v. 208, n. 1, p. 33-38, 1999.

[2] ANDERSEN, O. M. Anthocyanin occurrences and analyses. In: Oral presentation at International Workshop on Anthocyanins; Adelaide, Australia; April 17-19, 2002 .

[3] BERARDINI, N.; FEZER, R.; CONRAD, J.; BEIFUSS, U.; CARLE, R.; SCHIEBER, A. Screening of mango (Mangifera indica L.) cultivars for their contents of flavonol O- and xanthone C-glycosides, anthocyanins, and pectin. Journal of Agricultural and Food Chemistry, v. 53, n. 5, p. 1563-1570, 2005.

[4] BOYLES, M. J.; WROLSTAD, R. E. Anthocyanin composition of red raspberry juice: influences of cultivar, processing, and environmental factors. Journal of Food Science, v. 58, n. 5, p. 1135-1141, 1993.

[5] BRAVO, L. Polyphenols: chemistry, dietary sources, metabolism, and nutritional significance. Nutrition Reviews, v. 56, n. 11, p. 317-333, 1998.

[6] BURNS, J.; GARDNER, P. T., O'NEIL, J.; CRAWFORD, S.; MORECROFT, I.; McPHAIL, D. B.; LISTER, C.; MATTHEWS, D.; MacLEAN, M. R.; LEAN, M. E. J.; DUTHIE, G. G.; CROZIER, A. Relationship among antioxidant activity, vasodilatation capacity, and phenolic content of red wines. Journal of Agricultural and Food Chemistry, v. 48, n. 2, p. 220-230, 2000.

[7] CHAOVANALIKIT, A.; THOMPSON, M. M.; WROLSTAD, R. E. Characterization and quantification of anthocyanins and polyphenolics in blue honeysuckle (Lonicera caerulea L.). Journal of Agricultural and Food Chemistry, v. 52, n. 4, p. 848-852, 2004.

[8] COSTA, C. T.; HORTON, D.; MARGOLIS, S. A. Analysis of anthocyanins in foods by liquid chromatography, liquid chromatography-mass spectrometry and capillary electrophoresis. Journal of Chromatography A, v. 881, n. 1-2, p. 403-410, 2000.

[9] DONNER, H.; GAO, L.; MAZZA, G. Separation and characterization of simple and malonylated anthocyanins in red onions, Allium cepa L. Food Research International, v. 30, n. 8, p. 637-643, 1997.

[10] DRUST, R. W.; WROLSTAD, R. E. Separation and characterization of anthocyanins by HPLC. In: WROLSTAD, R. E. (Ed.) Current Protocols in Food Analytical Chemistry. New York: John Wiley \& Sons, 2001. p. Fl 3.1-3.13.

[11] ESPÍN, J. C.; SOLER-RIVAS, C.; WICHERS, H. J.; GARCÍA-VIGUERA, C. Anthocyanin-based natural colorants: a new source of antiradical activity for foodstuff. Journal of Agricultural and Food Chemistry, v. 48, n. 5, p. 1588-1592, 2000.

[12] GAO, L.; MAZZA, G. Characterization of acetylated anthocyanins in lowbush blueberries. Journal of Liquid Chromatography, v. 18, n. 2, p. 245-259, 1995.

[13] GiUSTI, M. M.; GHANADAN, H.; WROLSTAD, R. E. Elucidation of the structure and conformation of red radish (Raphanus sativus) anthocyanins using one- and two-dimensional nuclear magnetic resonance techniques. Journal of Agricultural and Food Chemistry, v. 46, n. 12, p. 4858-4863, 1998.

[14] GIUSTI, M. M.; RODRÍGUEZ-SAONA, L. E.; GRIFFIN, D.; WROLSTAD, R. E. Electrospray and tandem mass spectroscopy as tools for anthocyanin characterization. Journal of Agricultural and Food Chemistry, v. 47, n. 11, p. 4657-4664, 1999.

[15] HANAMURA, T.; HAGIWARA, T.; KAWAGISHI, H. Structural and functional characterization of polyphenols isolated from acerola (Malpighia emarginata DC.) fruit. Bioscience Biotechnology and Biochemistry, v. 69, n. 2, p. 280-286, 2005.

[16] HARRIS, R. S. Effects of agricultural practices on the composition of foods. In: HARRIS, R. S; KARMAS, E. Nutritional evaluation of food processing, Westport: The Avi Publishing Company, Inc., 1977. p. 33-57.

[17] ICHIYANAGI, T.; KASHIWADA, Y.; IKESHIRO, Y.; HATANO, Y.; SHIDA, Y.; HORIE, M.; MATSUGO, S.; KONISHI, T. Complete assignment of bilberry (Vaccinium myrtillus L.) anthocyanins separated by capillary zone electrophoresis. Chemical \& Pharmaceutical Bulletin, v. 52, n. 2, p. 226-229, 2004.

[18] JAYAPRAKASAM, B.; VAREED, S. K.; OLSON, L. K.; NAIR, M. G. Insulin secretion by bioactive anthocyanins and anthocyanidins present in fruits. Journal of Agricultural and Food Chemistry, v. 53, n. 1, p. 28-31, 2005.

[19] KÄHKÖNEN, M. P.; HEINONEN, M. Antioxidant activity of anthocyanins and their aglycons. Journal of Agricultural and Food Chemistry, v.51, n. 3, p. 628-633, 2003.

[20] KALLITHRAKA, S.; MOHDALY, A. A-A.; MAKRIS, D. P.; KEFALAS, P. Determination of major anthocyanin pigments in Hellenic native grapes varieties (Vitis vinifera sp.): association with antiradical activity. Journal of Food Composition and Analysis, v. 18, n. 5, p. 375-386, 2005.

[21] LIMA, V. L. A. G.; MÉLO, E. A.; MACIEL, M. I. S.; LIMA, D. E. S. Avaliação do teor de antocianinas em polpa de acerola congelada proveniente de frutos de 12 diferentes aceroleiras (Malpighia emarginata DC.). Ciência e Tecnologia de Alimentos, v. 23, n. 1, p. 101-103, 2003.

[22] LONGO, L.; VASAPOLLO, G.; RESCIO, L. Identification of anthocyanins in Rhamnus alaternus L. berries. Journal of Agricultural and Food Chemistry, v. 53, n. 5, p. 1723-1727, 2005.

[23] LONGO, L.; VASAPOLLO, G. Determination of anthocyanins in Ruscus aculeatus L. berries. Journal of Agricultural and Food Chemistry, v. 53, n. 2, p. 475-479, 2005.

[24] MACHEIX, J.-J.; FLEURIT, A.; BILlOT, J. Fruit Phenolics. Boca Raton: CRC Press, 1990.

[25] MATSUI, T.; EBUCHI, S.; KOBAYASHI, M.; FUKUI, K.; SUGITA, K.; TERAHARA, N.; MATSUMOTO, K. Antihyperglycemic effect of diacylated anthocyanin derived from Ipomoea batatas cultivar Ayamurasaki can be achieved through the $\alpha$-glucosidase inhibitory action. Journal of Agricultural and Food Chemistry, v. 50, n. 25, p. 7244-7248, 2002.

[26] MOZET, B.; TREBŠE, P.; HRIBAR, J. Determination and quantitation of anthocyanins and hidroxycinnamic acids in different cultivars of sweet cherries (Prunus avium L.) from Nova Gorica Region (Slovenia). Food 
Technology and Biotechnology, v. 40, n. 3, p. 207-212, 2002.

[27] NAKAISHI, H.; MATSUMOTO, H.; TOMINAGA, S.; HIRAYAMA, M. Effects of black currant anthocyanoside intake on dark adaptation and VDT work-induced transient refractive alteration in healthy humans. Alternative Medicine Review, v. 5, n. 6, p. 553-562, 2000.

[28] NYMAN, N. A., KUMPULAINEN, J. T. Determination of anthocyanidins in berries and red wine by high-performance liquid chromatography. Journal of Agricultural and Food Chemistry, v. 49, n. 9, p. 4183-4187, 2001.

[29] OLIVEIRA, J. R. P.; SOARES FILHO, W. S. Situação da cultura da acerola no Brasil e ações da Embrapa Mandioca e Fruticultura em recursos genéticos e melhoramento. In: QUEIRÓ, M. A.; GOEDERT, C. O.; RAMOS, S. R. R. (Eds). Recursos Genéticos e Melhoramento de Plantas para o Nordeste Brasileiro. Petrolina: Embrapa Semi-Árido; Brasília: Embrapa Recursos Genéticos e Biotecnologia, 1999. Disponível em: <http://www.cpatsa.embrapa.br/servicos/catalogo/ livrorg/termos.html>. Acesso em: 27 set. 2005.

[30] ROBARDS, K.; PRENZLER, P. D.; TUCKER, G.; SWATSITANG, P.; GLOVER, W. Phenolic compounds and their role in oxidative processes in fruits. Food Chemistry, v. 66, n.4, p. 401-436, 1999.

[31] RODRIGUEZ-SAONA, L.E.; WROLSTAD, R.E. Extraction, isolation, and purification of anthocyanins. In: WROLSTAD, R. E. (Ed.) Current Protocols in Food Analytical Chemistry New York: John Wiley \& Sons, 2001. p.Fl 1.1-1.11.

[32] SEERAM, N. P.; MOMIN, R. A.; NAIR, M. G.; BOURQUIN, L. D. Cyclooxygenase inhibitory and antioxidant cyanidin glycosides in cherries and berries. Phytomedicine, v. 8, n. 5, p. 362-369, 2001.

[33] SILVA, M. F. V. Efeito dos diferentes tratamentos e embalagens nas características da polpa de acerola e na determinação dos teores de ácido ascórbico e das antocianinas durante o armazenamento. Campinas, 1999, 224p. Tese (Doutorado em Tecnologia de Alimentos). Faculdade de Engenharia de Alimentos, Universidade Estadual de Campinas (UNICAMP).

[34] SIRIWOHARN, T.; WROLSTAD, R. E.; FINN, C. E.; PEREIRA, C. B. Influence of cultivar, maturity, and sampling on blackberry (Rubus L. Hybrids) anthocyanins, polyphenolics, and antioxidant properties. Journal of Agricultural and Food Chemistry, v. 52, n. 26, p. 8021-8030, 2004.

[35] STRACK, D.; WRAY, V. The anthocyanins. In: HARBORNE, J. B. (Ed.) The Flavonoids: advances in research since 1986. Boca Raton: Chapman \& Hall, 1994. p. 1-22.

[36] VENDRAMINI, A. L. A.; TRUGO, L. C. Phenolic compounds in acerola fruit (Malpighia punicifolia, L.). Journal of the Brazilian Chemical Society, v. 15, n. 5, p. 664-668, 2004.

[37] WROLSTAD, R. E. Anthocyanins pigments - bioactivity and coloring properties. Journal of Food Science, v. 69, n. 5, p. C419-C421, 2004.

[38] WU, X.; PRIOR, R. L. Systematic identification and characterization of anthocyanins by HPLC-ESI-MS/MS in common foods in the United States: fruits and berries. Journal of Agricultural and Food Chemistry, v. 53, n. 7, p. 2589-2599, 2005.

[39] ZHANG, Z.; KOU, X.; FUGAL, K.; MCLAUGHLIN, J. Comparison of HPLC methods for determination of anthocyanins and anthocyanidins in bilberry extracts. Journal of Agricultural and Food Chemistry, v. 52 , n. 4, p. 688-691, 2004.

[40] ZHANG, Y.; VAREED, S. K.; NAIR, M. G. Human tumor cell growth inhibition by nontoxic anthocyanidins, the pigments in fruits and vegetables. Life Sciences, v. 76 , n. 13, p. 1465-1472, 2005. 